\title{
Clinically Worsening Chronic Thromboembolic Pulmonary Hypertension by Riociguat After Balloon Pulmonary Angioplasty
}

\author{
Shun Minatsuki, ${ }^{1}$ MD, Masaru Hatano, ${ }^{2}$ MD, Arihiro Kiyosue, ${ }^{1} \mathrm{MD}$, Akihito Saito, ${ }^{1} \mathrm{MD}$, \\ Hisataka Maki, ${ }^{1} \mathrm{MD}$, Eiki Takimoto,${ }^{3} \mathrm{MD}$ and Issei Komuro, ${ }^{1} \mathrm{MD}$
}

\begin{abstract}
Summary
Riociguat, a soluble guanylate cyclase stimulator, induces pulmonary artery dilatation through blood flow and is effective in treating chronic thromboembolic pulmonary hypertension (CTEPH). There are two types of vasculopathies in CTEPH based upon its location, in other words, proximal or distal to the thrombus-medicated obstruction. Distal vasculopathy is characterized by intrapulmonary shunts due to diminished blood flow. While other therapeutic interventions for CTEPH including pulmonary endarterectomy and balloon pulmonary angioplasty achieve reperfusion to the distal vasculopathy vessels, the effects of riociguat on distal vasculopathy vessels remain undetermined. Herein, we describe a case of a 66-year-old woman who exhibited deterioration of mean pulmonary artery pressure and exercise tolerance after a 4-month treatment with riociguat. She received balloon pulmonary angioplasty prior to riociguat administration. Her lung perfusion scintigraphy and pulmonary angiography findings did not change over the course of treatment. Notably, after the discontinuation of riociguat, her clinical values returned to their levels prior to riociguat administration. Her intrapulmonary shunt ratio followed a similar course as her hemodynamic status. We demonstrate that riociguat can deteriorate hemodynamic status, which may mediate the dilatation of intrapulmonary shunts. We should perform close monitoring of symptoms and hemodynamic status after riociguat administration, especially in patients in whom the reperfused DVs occurred due to invasive treatment.
\end{abstract}

(Int Heart J 2018; 59: 1186-1188)

Key words: Distal vasculopathy, Intrapulmonary shunt, Inappropriate vasodilation

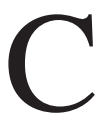
hronic thromboembolic pulmonary hypertension (CTEPH) occurs when organized thrombi restrict pulmonary artery blood flow. Pathologically, CTEPH has two types of vasculopathies that are based upon the location of the obstruction. ${ }^{1)}$ Notably, vessels distal to the site of obstruction exhibit diminished blood flow, intrapulmonary shunts, and endothelial cell abnormalities $^{1)}$ (distal vasculopathy: DV). Riociguat, a soluble guanylate cyclase stimulator, has been approved for inoperable or persistent/recurrent CTEPH. ${ }^{2,3)}$ It functions by increasing the level of cyclic guanosine monophosphate, thereby resulting in vasorelaxation. ${ }^{4)}$ These effects are exerted in the presence of blood flow. Although riociguat may be used in combination with other therapeutic interventions such as BPA, the efficacy of riociguat in reperfused DVs remains to be established. Here, we present a case in which treatment with riociguat worsened an intrapulmonary shunt and caused deterioration of the mean pulmonary artery pressure (mPAP) and exercise tolerance.

\section{Case Report}

A 66-year-old woman was transferred to our hospital for the treatment of severe right heart failure due to CTEPH. She had no history of lung disease or smoking. Upon admission, her condition was World Health Organization functional class IV. Right heart catheterization revealed a severely compromised hemodynamic status and oxygenation was markedly deteriorated in her initial right heart catheterization test (Table). Thus, she required mechanical ventilation with intratracheal intubation to maintain arterial oxygenation. She underwent 6 sessions of balloon pulmonary angioplasty (BPA) and discontinued mechanical ventilation. There was no evidence of lung bleeding induced by BPA. Before discharge, a right heart catheterization test revealed an improvement in her hemodynamic status (Table), and she was started on riociguat (3 $\mathrm{mg} /$ day). The riociguat dose was titrated up to $7.5 \mathrm{mg}$ /day without any adverse events. A follow-up right heart catheterization test was performed 4 months later and revealed a worsening hemodynamic status and 6-minute walk dis-

From the ${ }^{1}$ Department of Cardiovascular Medicine, Graduate School of Medicine, The University of Tokyo, Tokyo, Japan, ${ }^{2}$ Department of Therapeutic Strategy for Heart Failure, Graduate School of Medicine, The University of Tokyo, Tokyo, Japan and ${ }^{3}$ Department of Advanced Translational Research and Medicine in Management of Pulmonary Hypertension, Graduate School of Medicine, The University of Tokyo, Tokyo, Japan.

Address for correspondence: Shun Minatsuki, MD, Department of Cardiovascular Medicine, Graduate School of Medicine, The University of Tokyo, 7-3-1 Hongo, Bunkyo-ku, Tokyo 113-8655, Japan. E-mail: sminatsuki-tky@umin.ac.jp

Received for publication November 17, 2017. Revised and accepted December 16, 2017

Released in advance online on J-STAGE August 29, 2018.

doi: 10.1536/ihj.17-660

All rights reserved by the International Heart Journal Association. 
Table. The Patient's Hemodynamic Status and Exercise Tolerance over the Course of the Disease

\begin{tabular}{|c|c|c|c|c|}
\hline & Before BPA & $\begin{array}{c}\text { Before riociguat } \\
\text { administration }\end{array}$ & $\begin{array}{l}\text { After riociguat } \\
\text { administration }\end{array}$ & $\begin{array}{l}\text { After riociguat } \\
\text { discontinuation }\end{array}$ \\
\hline $\mathrm{FiO}_{2}$ & 0.36 & 0.21 & 0.21 & 0.21 \\
\hline Medication & $\begin{array}{c}\text { Bosentan } 125 \mathrm{mg} \\
\text { Beraprost } 120 \mu \mathrm{g} \\
\text { Sildenafil } 60 \mathrm{mg} \\
\text { Furosemide } 40 \mathrm{mg} \\
\text { Spironolactone } 50 \mathrm{mg} \\
\text { Dobutamin } 3 \gamma \\
\text { Milrinone } 0.14 \gamma\end{array}$ & $\begin{array}{r}\text { Warfarin } 2.75 \mathrm{mg} \\
\text { Furosemide } 20 \mathrm{mg} \\
\text { Spironolactone } 50 \mathrm{mg}\end{array}$ & $\begin{array}{l}\text { Riociguat } 7.5 \mathrm{mg} \\
\text { Warfarin } 2.75 \mathrm{mg}\end{array}$ & Warfarin $2.5 \mathrm{mg}$ \\
\hline Heart rate $(\mathrm{bpm})$ & 121 & 89 & 88 & 74 \\
\hline $\mathrm{BNP}(\mathrm{pg} / \mathrm{mL})$ & 1228.2 & 26.5 & 18.2 & 21.8 \\
\hline mRAP $(\mathrm{mmHg})$ & 17 & 3 & 6 & 4 \\
\hline $\operatorname{PAP}(\mathrm{s} / \mathrm{d} / \mathrm{m})(\mathrm{mmHg})$ & $65 / 41 / 52$ & $46 / 12 / 24$ & $62 / 12 / 37$ & $48 / 12 / 26$ \\
\hline $\mathrm{CO}(\mathrm{L} / \mathrm{minute})$ & 2.9 & 3.6 & 5.3 & 3.7 \\
\hline PVR (dynes) & 1231 & 399 & 440 & 368 \\
\hline $\mathrm{SaO}_{2}(\%)$ & 91.4 & 96.4 & 93.5 & 95.4 \\
\hline $\mathrm{SvO}_{2}(\%)$ & 54.2 & 70.5 & 76.0 & 70.6 \\
\hline $\mathrm{A}-\mathrm{aDO}_{2}$ (Torr) & 166.4 & 20.5 & 36.4 & 17.1 \\
\hline Intra pulmonary shunt ratio & N/A & 15.2 & 23.9 & 12.7 \\
\hline 6 minute walk distance $(\mathrm{m})$ & N/A & 430 & 400 & 460 \\
\hline
\end{tabular}

$\mathrm{FiO}_{2}$ indicates fraction of inspiratory oxygen; BNP, B-type natriuretic peptide; mRAP, mean pressure of right atrium; PAP, pulmonary artery pressure; $\mathrm{CO}$, cardiac output; PVR, pulmonary vascular resistance; $\mathrm{SaO}_{2}$, arterial oxygen saturation; $\mathrm{SvO}$, mixed venous oxygen saturation; $\mathrm{A}-\mathrm{aDO}_{2}$, alveolar-arterial oxygen difference; and BPA, balloon pulmonary angioplasty.
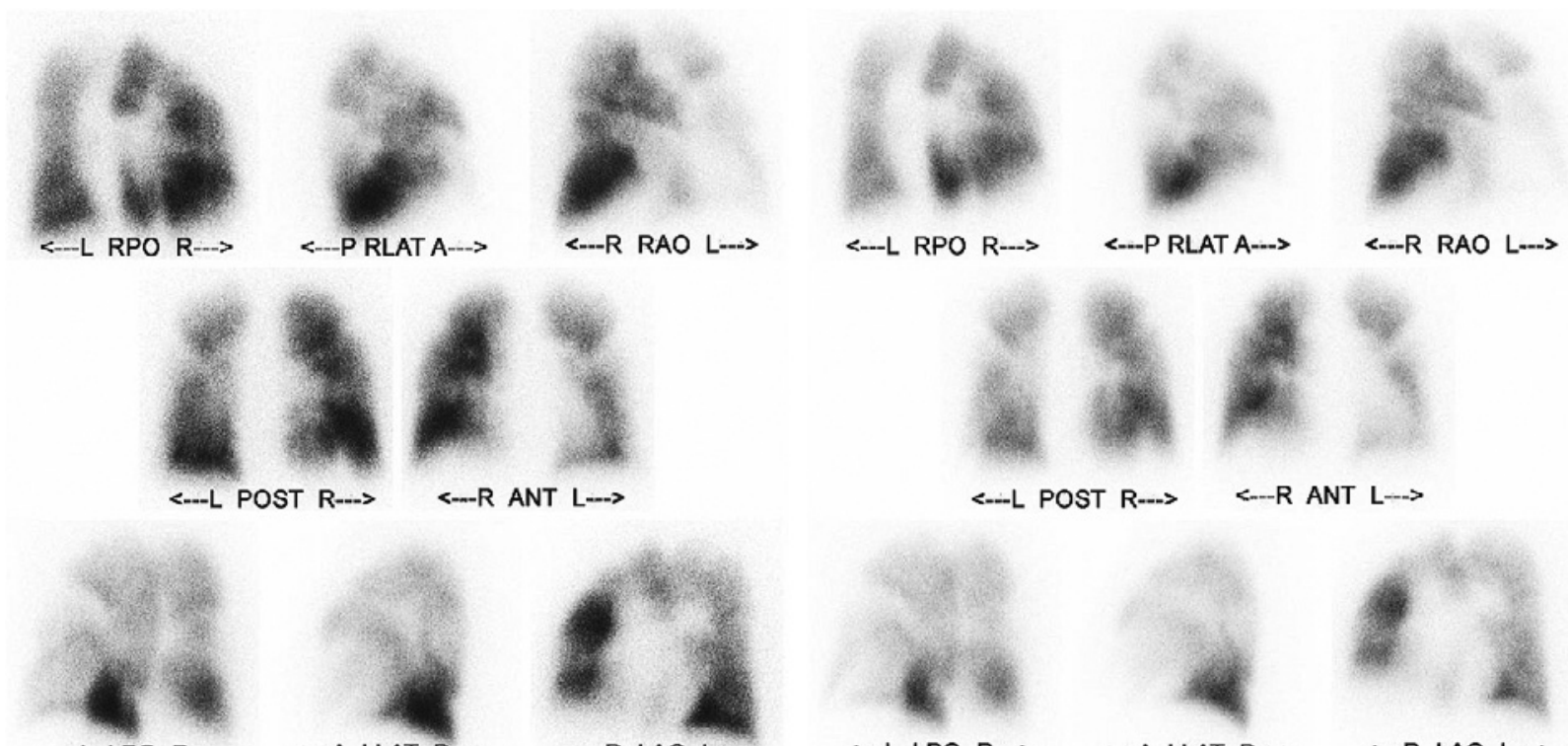

$<--$ L LPO R->
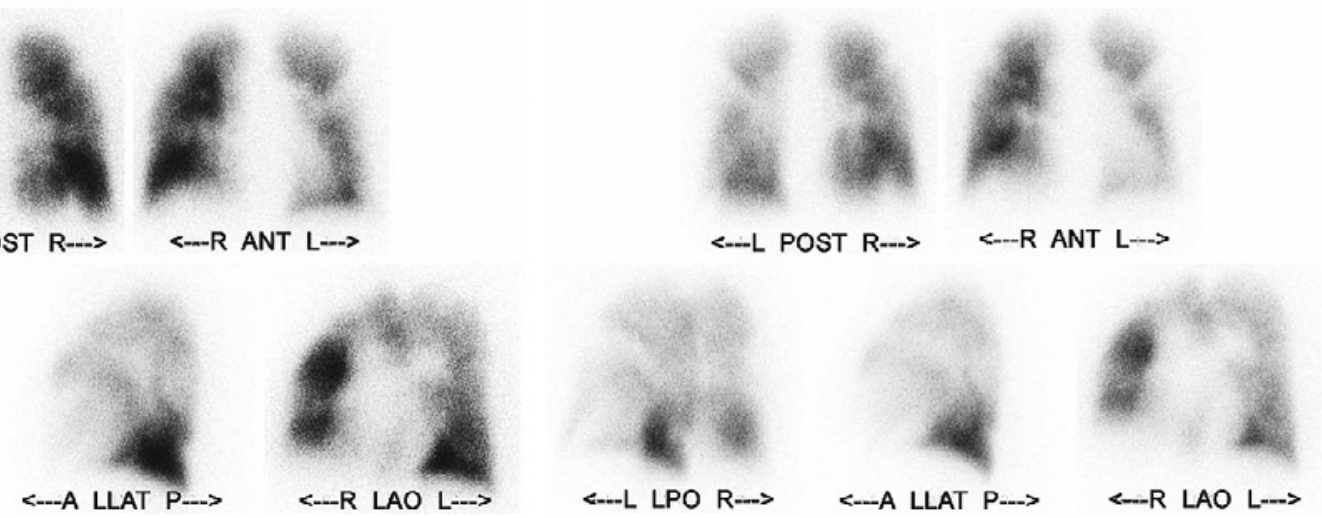

Figure. Scintigram before (left) and after (right) the administration of riociguat.

tance (Table), though she had no symptoms in her daily life. At that time, we calculated the pulmonary shunt ratio using the amount of loading oxygen, which was found to have worsened. Additionally, her cardiac output was elevated. Meanwhile, the lung perfusion scintigraphy and pulmonary angiography findings remained unchanged from 4 months prior (Figure). We discontinued treatment with riociguat and performed follow-up right heart catheterization 1 month later. Her hemodynamic status, 6minute walk distance, and shunt ratio returned to their pre-discharge values. Since then, the patient has main- tained a good hemodynamic status and is symptom-free in daily life.

\section{Discussion}

CTEPH has two vasculopathies that are divided based upon location. Proximal vasculopathy, which occurs within vessels proximal to the site of obstruction, includes vascular remodeling, such as pulmonary arterial hypertension due to blood overflow and excessive pressure load. ${ }^{1)}$ The second vasculopathy is DV, which exhibits dimin- 
ished blood flow and intrapulmonary shunts. ${ }^{1)}$ Riociguat is an oral drug whose efficacy requires the presence of blood flow. Most patients included in previous studies showing the efficacy of riociguat were inoperable. ${ }^{4)}$ Thus, the efficacy of riociguat is chiefly mediated by its impact on proximal vasculopathy. Meanwhile, treatments for CTEPH, such as pulmonary endarterectomy and BPA, improve perfusion to DVs. ${ }^{5}$ After such treatment, DV reperfusion occurs; however, the efficacy of riociguat in reperfused DVs is unclear.

We experienced another case of a 61-year-old woman with CTEPH whose clinical course worsened with riociguat treatment. Her hemodynamic status and oxygenation level improved after riociguat administration (7.5 $\mathrm{mg}$ /day) and two sessions of BPA (mPAP $50 \rightarrow 37 \mathrm{mmHg}$, cardiac output $(\mathrm{CO}) 3.6 \rightarrow 3.9 \mathrm{~L} /$ minute, arterial oxygen saturation $\left.\left(\mathrm{SaO}_{2}\right) 90.4 \rightarrow 93.3 \%\right)$. However, 3 months later, her mPAP was incidentally elevated to $44 \mathrm{mmHg}$ along with an inappropriate elevation of $\mathrm{CO}(6.5 \mathrm{~L} /$ minute $)$ and a decrease in $\mathrm{SaO}_{2}(89.8 \%)$. Her hemodynamic status and oxygenation returned to pre-exacerbation levels (mPAP 32 $\mathrm{mmHg}, \mathrm{CO} 4.3 \mathrm{~L} / \mathrm{min}, \mathrm{SaO}_{2} 91.3 \%$ ) 1 week after the discontinuation of riociguat, as had occurred in the present case.

Both patients exhibited elevated mPAP after riociguat administration. Because of the inappropriate $\mathrm{CO}$ elevation, pulmonary vascular resistance did not significantly change immediately after riociguat administration. BPA treatment, which results in reperfusion to the DVs, appeared to dilate the pulmonary shunt. This may have exacerbated the intrapulmonary shunt ratio, which resulted in hemodynamic deterioration and inappropriate ventilation-perfusion distribution.

The treatment of CTEPH, including with riociguat and BPA, has improved dramatically. ${ }^{4-7)}$ It has been thoroughly documented that riociguat is an effective drug for CTEPH. Thus, the hybrid of riociguat and invasive methods is becoming a mainstream therapeutic method in
CTEPH, however, the use of riociguat can result in the deterioration of hemodynamic status and exercise tolerance in rare cases. These two outcomes can be the result of inappropriate dilatation of the pulmonary shunt due to riociguat use. However, the characteristics of the patients susceptible to these adverse effects of riociguat use remain unclear. We should perform close monitoring of symptoms and hemodynamic status after riociguat administration, especially in patients in whom the reperfused DVs occurred due to invasive treatment.

\section{Disclosures}

Conflicts of interest: None.

\section{References}

1. Galie N, Kim NH. Pulmonary microvascular disease in chronic thromboembolic pulmonary hypertension. Proc Am Thorac Soc 2006; 3: 571-6.

2. Kim NH, D’Armini AM, Grimminger F, et al. Haemodynamic effects of riociguat in inoperable/recurrent chronic thromboembolic pulmonary hypertension. Heart 2017; 103: 599-606.

3. Pepke-Zaba J, Ghofrani HA, Hoeper MM. Medical management of chronic thromboembolic pulmonary hypertension. Eur Respir Rev 2017; 26: 160107.

4. Ghofrani HA, D'Armini AM, Grimminger F, et al. Riociguat for the treatment of chronic thromboembolic pulmonary hypertension. N Engl J Med 2013; 369: 319-29.

5. Minatsuki S, Hatano M, Maki H, et al. The structure of a chronic total occlusion and its safe treatment in a patient with chronic thromboembolic pulmonary hypertension. Int Heart $\mathrm{J}$ 2017; 58: 824-7.

6. Ogawa A, Matsubara H. Balloon pulmonary angioplasty: a treatment option for inoperable patients with chronic thromboembolic pulmonary hypertension. Frontiers Cardiovasc Med 2015; 2: 4 .

7. Minatsuki S, Kiyosue A, Saito A, et al. Effectiveness of nitroglycerin in managing subacute lung bleeding induced by balloon pulmonary angioplasty. Int Heart J 2018; 59: 899-901. 